\title{
TLR4 mediates inflammation and hepatic fibrosis induced by chronic intermittent hypoxia in rats
}

\author{
ZHI-PENG LIN ${ }^{1}$, HUI-LI LIN ${ }^{2}$, XUE-PING YU ${ }^{1}$, YI-JUAN ZHENG ${ }^{1}$ and SI-YU CHENG ${ }^{1}$ \\ ${ }^{1}$ Department of Infectious Diseases, The First Quanzhou Affiliated Hospital of Fujian Medical University; \\ ${ }^{2}$ Department of Cardiology, The Second Affiliated Hospital of Fujian Medical University, \\ Quanzhou, Fujian 362000, P.R. China
}

Received December 18, 2018; Accepted March 5, 2020

DOI: $10.3892 / \mathrm{mmr} .2020 .11134$

\begin{abstract}
Obstructive sleep apnea syndrome (OSAS) is a common and complex disorder that is associated with liver injury. Moreover, previous studies have revealed that chronic intermittent hypoxia $(\mathrm{CIH})$ is associated with the development of non-alcoholic fatty liver disease and hepatic fibrosis. However, the underlying molecular mechanisms remain largely unknown. The present study aimed to investigate whether chronic intermittent hypoxia induced hepatic fibrosis, in addition to determining its underlying mechanisms, in CIH model rats using immunohistochemistry, western blotting and reverse transcription-quantitative PCR. The present results suggested that $\mathrm{CIH}$ caused hepatic fibrosis and increased the expression levels of interleukin (IL)-1 $\beta$, IL-8, monocyte chemotactic-1, tumor necrosis factor- $\alpha$, intercellular adhesion molecule- 1 and vascular cell adhesion molecule-1 in the liver; these conditions could be reversed by Toll-like receptor 4 (TLR4) short hairpin RNA lentivirus treatment. Moreover, immunohistochemistry and western blotting results indicated that TLR4 and NF- $\mathrm{BB}$ expression levels were significantly increased in the $\mathrm{CIH}$ and CIH-TLR4 empty vector lentivirus group. However, protein expression levels of TLR4, NF- $\kappa \mathrm{B}$, inhibitor of $\mathrm{NF}-\kappa \mathrm{B}$ and phosphorylated-mitogen-activated protein kinase (MAPK)-1 in the hypoxia/reoxygenation group were significantly higher compared with the control group $(\mathrm{P}<0.05)$, and these results were reversed by the MAPK inhibitor U0126 in vitro. Collectively, the present preliminary results suggested that inflammation and the TLR4/NF- $\mathrm{B} / \mathrm{MAPK}$ signaling pathway may be involved in $\mathrm{CIH}$-induced liver fibrosis.
\end{abstract}

Correspondence to: Dr Hui-Li Lin, Department of Cardiology, The Second Affiliated Hospital of Fujian Medical University, 34 Zhongshan Road, Quanzhou, Fujian 362000, P.R. China E-mail: huililin@yeah.net

Key words: obstructive sleep apnea syndrome, chronic intermittent hypoxia, inflammation, liver fibrosis, Toll-like receptor 4

\section{Introduction}

Obstructive sleep apnea syndrome (OSAS) is a common and complex disorder consisting of complete or partial upper airway obstruction and sleep fragmentation, affecting $>4 \%$ of the world's general population and $30-45 \%$ of patients with obesity (1-3). As a result of upper airway collapse, OSAS causes repeated nocturnal hypoxia and alternate episodes of normoxia, such chronic intermittent hypoxia (CIH) during sleep, resembling the pathophysiologic mechanisms in ischemia/reperfusion multi-organ injury, which can trigger cardiovascular morbidity, lung injury and chronic kidney disease (4-7). Moreover, previous studies have reported that $\mathrm{CIH}$ contributes to the pathogenesis of non-alcoholic fatty liver disease and exacerbates liver fibrogenesis (2-8). However, the underlying mechanisms of hepatic fibrosis in patients with OSAS are not fully understood.

Epidemiological studies and clinical investigations have shown that OSAS is a type of chronic and mild systemic inflammatory response disease $(8,9)$. For example, inflammatory factors such as interleukin (IL)- $\beta$, IL-6, IL-18, tumor necrosis factor- $\alpha$ (TNF- $\alpha$ ), interferon- $\gamma$ and C-reactive protein (CRP) can be detected at high levels in the blood of patients with OSAS (10,11). Furthermore, Ryan and McNicholas (11) revealed a significant positive association between serum TNF- $\alpha$ levels and OSAS severity, with higher serum levels of TNF- $\alpha$ in patients with OSAS compared with non-OSAS subjects. In addition, Drager et al (12) reported that the expression of CRP declined in adult patients with OSAS after effective treatment with continuous positive airway pressure. Therefore, these findings suggest that $\mathrm{CIH}$ may be associated with inflammation and may contribute to the increased incidence of liver fibrogenesis in patients with OSAS. However, although several studies have closely linked CIH to systemic inflammation, the mechanism of this association has not been fully established.

Recent studies have shown that inflammation is associated with innate immune activation, including that involving Toll-like receptors (TLRs) and its underlying signal pathway $(13,14)$. TLRs belong to a class of receptors with well-known pattern recognition, and can accurately perceive pathogens and bacterial-derived molecules (15). Moreover, activation of TLRs can lead to inflammatory responses, thus 
increasing the production of proinflammatory cytokines (16). TLR4, a typical representative of TLRs, mediates both innate and adaptive immune responses and plays an essential role in promoting inflammation activation $(14,17)$. It has also been reported that TLR4 can trigger the main adaptor protein, myeloid differentiation factor 88 (MyD88)-dependent pathway, leading to rapid activation of the classical $N F-\kappa B$ and mitogen-activated protein kinase (MAPK) signaling pathway, which upregulates the transcription and translation of proinflammatory genes, such as IL-1 $\beta$, IL-6, IL-8, IL-18 and TNF- $\alpha(18,19)$. Previous studies have revealed that $\mathrm{CIH}$ can increase mRNA and protein expression levels of TLR4 in the heart and hypothalamus of rats, and can induce myocardial remodeling and neuronal cell damage in hippocampus, which may be involved in CIH-induced inflammation $(20,21)$. Furthermore, it has been shown that TLR4 expression in the serum of patients with OSAS is significantly increased compared with healthy individuals (22). However, the relationship between abnormal expression of TLR4 and the severity of liver injury in patients with OSAS has not been fully elucidated, and there is limited information on the downstream changes of TLR signal transduction after CIH exposure.

The aims of the present study were as follows: i) To investigate whether $\mathrm{CIH}$ exposure can induce similar liver fibrosis pathological changes in rats as in patients with OSAS; ii) to examine whether $\mathrm{CIH}$ affects proinflammatory cytokine production in the liver; and iii) to identify the underlying molecular mechanisms and signaling pathways that may be involved in $\mathrm{CIH}$-induced liver fibrosis.

\section{Materials and methods}

Animals. A total of 24 adult male Sprague-Dawley rats (age, 9 weeks; weight, $200 \pm 10 \mathrm{~g}$ ) were purchased from Shanghai Xipuer-Bikai Laboratory Animal Co., Ltd. Rats were fed and housed in a standard pathogen-free environment with a $12 \mathrm{~h}$ light/12 h dark cycle (7:00 a.m. lights on and 7:00 p.m. lights off automatically). The rats were provided with special compound diet and sterilization water ad libitum. Room temperature and humidity were controlled at $\sim 23 \pm 2^{\circ} \mathrm{C}$ and $50 \pm 10 \%$, respectively. All animal procedures were approved by the Ethical Committee of Experimental Animals of Fujian Medical University and The Second Affiliated Hospital of Fujian Medical University. Animal experiments were carried out in accordance with animal welfare requirements and the Guidelines for the Care and Use of Laboratory Animals published by the P.R. China Ministry of Health (January 25, 1998) (23).

CIH animal model construction and experimental design. Rats were divided into the control (Con) group $(n=6), \mathrm{CIH}$ group (n=6), CIH + TLR4 short hairpin (sh)RNA lentivirus group (CIH-TLR4) $(\mathrm{n}=6)$ and $\mathrm{CIH}+$ TLR4 empty vector lentivirus group (CIH-Vector) $(\mathrm{n}=6)$. Firstly, $10 \mu \mathrm{l}\left(2 \times 10^{6}\right.$ TU/ $\mu 1$ ) TLR4 shRNA lentivirus (5'-GATCCGCACTCTT GATTGCAGTTTCATTCAAGAGATGAAACTGCAATCA GAGTGCTTTTTTG-3'), scrambled TLR4 shRNA (5'-GTA GCTAAATGATGAACAACATGGCCTTATATCTCCTAGT AATCTTCGTGGCTTCGTAGTGAT-3') and TLR4 shRNA empty vector lentivirus (Shanghai GenePharma Co., Ltd.) were separately injected into the rats of the CIH-TLR4 group, Con group or CIH-Vector group, respectively, via the tail vein. After 1 week of recovery, each rat was placed in a transparent plastic container (length, $40 \mathrm{~cm}$; width, $30 \mathrm{~cm}$; height, $18 \mathrm{~cm}$ ), which was connected to a device for controlling $\mathrm{O}_{2}$ concentration and pressure (Puhe Biotechnology). For consistency with the rat sleep cycle, the experimental time was set from 8:00 to 20:00 every day. Rats in the Con group were supplied with air, whereas those in the CIH, CIH-TLR4 and CIH-Vector group were subjected to intermittent hypoxia treatment, by injecting $95 \% \mathrm{~N}_{2}$ for $20 \mathrm{sec}$ to rapidly reduce $\mathrm{O}_{2}$ concentration to $7.4-7.8 \%$. Container pressure was reduced to $600 \mathrm{mmHg}$. The set-up was held for $12 \mathrm{sec}$, and then injected with air for $28 \mathrm{sec}$ to recover $\mathrm{O}_{2}$ concentration to $21 \%$ and achieve normal air pressure. The entire experiment lasted for 4 weeks.

RNA isolation and reverse transcription-quantitative PCR (RT-qPCR). After CIH modeling, all rats were anesthetized (sodium pentobarbitone, $40 \mathrm{mg} / \mathrm{kg}$ ) and sacrificed by exsanguination. A small piece of liver in each group was quickly cut and removed. RNA was extracted using a RNeasy Mini kit (Qiagen $\mathrm{GmbH}$ ) following the manufacturer's protocol and stored at $-80^{\circ} \mathrm{C}$ for subsequent use. Total RNA was reverse transcribed into cDNA at $42^{\circ} \mathrm{C}$ for $30 \mathrm{~min}$ using a QuantiTect Reverse Transcription kit (Qiagen $\mathrm{GmbH}$ ), according to the manufacturer's protocol. qPCR reactions were performed using iTaq SYBR Green kits (Toyobo Life Science), according to the manufacturer's protocol. All reactions were carried out in duplicate and the cycles were run on a Bio-Rad CFX96 RT system (Bio-Rad Laboratories, Inc.). The following thermocycling conditions were used: Initial denaturation at $95^{\circ} \mathrm{C}$ for $30 \mathrm{sec}$; followed by 35 cycles of annellation at $58^{\circ} \mathrm{C}$ for $40 \mathrm{sec}$ and elongation at $72^{\circ} \mathrm{C}$ for $30 \mathrm{sec}$. The primers used were as follows: IL- $1 \beta$ forward, 5'-AGGAGAGACAAGCAA CGACAA-3' and reverse, 5'-GTTTGGGATCCACACTCT CCA-3'; IL-8 forward, 5'-ATGGCTGCTGAACCAGTAGA-3' and reverse, 5'-CTAGTCTTCGTTTTGAACAG-3'; monocyte chemotactic-1 (MCP-1) forward, 5'-TCCACCACTATGCAG GTCTC-3' and reverse, 5'-TGGACCCATTCCTTATTGGG-3'; TNF- $\alpha$ forward, 5'-AGAACTCCAGCCGGTGTCTGTG-3' and reverse, 5'-GTGGCAAATCGGCTGACGGTGT-3'; intercellular adhesion molecule-1 (ICAM-1) forward, 5'-GGC GTCCATTTACACCTATTA-3' and reverse, 5'-TTCCTTTTC TTCTCTTGCTTG-3'; vascular cell adhesion molecule-1 (VCAM-1) forward, 5'-AACTGCACGGTCCCTAAT-3' and reverse, 5'-AGATGGTGGGTTCTTTCG-3'; and $\beta$-actin forward, 5'-AGCCATGTACGTAGCCATCC-3' and reverse, 5'-ACCCTCATAGATGGGCACAG-3'. Expression levels were quantified using the $2^{-\Delta \Delta \mathrm{Cq}}$ method (24).

Tissue immunohistochemistry. After $\mathrm{CIH}$ modeling, all rats were anesthetized (sodium pentobarbitone, $40 \mathrm{mg} / \mathrm{kg}$ ) and sacrificed by exsanguination. A small piece of liver in each group was quickly cut and fixed with $10 \%$ formalin for $48 \mathrm{~h}$ at $4^{\circ} \mathrm{C}$. Samples were then embedded in paraffin and cut into tissue sections $(3-\mu \mathrm{m})$ using a tissue microtome. The deparaffinized tissue sections were subjected to hematoxylin and eosin (H\&E) staining, both for $10 \mathrm{~min}$ at room temperature, and observed under a light microscope (magnification, $x 400$ ) to obtain accurate pathological diagnosis. 
Different sections in each group were stained via standard Sirius red staining at room temperature for $30 \mathrm{~min}$ to observe and determine reactive fibrosis, along with its distribution as described previously (25). Liver tissues were blocked with $5 \%$ BSA (Toyobo Life Science) for $1 \mathrm{~h}$ at room temperature followed by incubation with IL-1 $\beta$ (Abcam; cat. no. ab9787), IL-8 (Santa Cruz Biotechnology, Inc.; cat. no. sc-376750), MCP-1 (Abcam; cat. no. ab25124), TNF- $\alpha$ (Abcam; cat. no. ab9755), ICAM-1 (Abcam; cat. no. ab171123), VCAM-1 (Abcam; cat. no. ab78712), TLR4 (Abcam; cat. no. ab95562) and NF- $\kappa \mathrm{B}$ antibodies (Abcam; cat. no. ab16502) diluted at 1:100 in PBS with 5\% BSA (Toyobo Life Science) at $4^{\circ} \mathrm{C}$ overnight. After washing with PBS three times, all tissue sections were incubated with horseradish peroxidase (HRP)-labeled secondary antibodies (Abcam; cat. nos. ab7090 and ab97040) diluted at 1:200 at room temperature for $30 \mathrm{~min}$ to assess liver inflammation in rats. The sections were then stained with 3'3-diaminobenzidine at room temperature for $15 \mathrm{~min}$ and the immune reaction results were observed by light microscopy (magnification, $x 400)$. The statistical results of these immunohistochemical images were analyzed by ImageJ version $1.52 \mathrm{t}$ software (National Institutes of Health).

Western blot analysis. Total protein of each group was isolated from the liver tissue by ultrasonic homogenization $(20 \mathrm{kHZ}$; $15 \mathrm{sec} ; 4^{\circ} \mathrm{C}$ ) in pre-cooled cell lysis solution (Sangon Biotech Co., Ltd.) containing protease and phosphatase inhibitors to inhibit protein degradation (Sangon Biotech Co., Ltd.). Then, cell lysate products were centrifuged at $10,000 \mathrm{x} \mathrm{g}$ for $30 \mathrm{~min}$ at $4^{\circ} \mathrm{C}$ in a refrigerated centrifuge. Total protein was quantified using the bicinchoninic acid assay method. Proteins (30 $\mu \mathrm{g} / \mu \mathrm{l} / \mathrm{lane})$ were separated by $10 \%$ SDS-PAGE in Tris-glycine-SDS buffer by vertical electrophoresis for $90 \mathrm{~min}$. After separation via electrophoresis, proteins were immediately transferred to prepared nitrocellulose (NC) membranes using a trans-blot transfer system (Sangon Biotech Co., Ltd.). NC membranes were blocked with 5\% skim milk diluted by $2 \%$ PBS (Sangon Biotech Co., Ltd.) for $60 \mathrm{~min}$ at room temperature and then incubated with primary anti-TLR4 (Abcam; cat. no. ab95562; 1:1,000), anti-inhibitor of NF- $\mathrm{B}$ (IкB; Abcam; cat. no. ab32518; 1:500), anti-MAPK-1 (Abcam; cat. no. ab32081; 1:500), anti-phosphorylated (p)-MAPK-1 (Abcam; cat. no. ab223500; 1:500), anti-NF- $\mathrm{B}$ p65 (Abcam; cat. no. ab16502; 1:500) and anti- $\beta$-actin (Abcam; cat. no. ab8226; 1:3,000) antibodies overnight at $4^{\circ} \mathrm{C}$. Then, the antibodies were diluted with 5\% BSA (Sangon Biotech Co., Ltd.) in TBS-0.1\% Tween-20. After washing with PBS three times, NC membranes were incubated with 5\% skim milk diluted HRP-conjugated secondary antibodies (cat. nos. D110261 and D110273, 1:1,000; Sangon Biotech Co., Ltd.) for $60 \mathrm{~min}$ at room temperature. The membrane blots were then visualized using a chemiluminescence instrument and enhanced chemiluminescence liquid kit (Pierce; Thermo Fisher Scientific, Inc.). Optical density value of each blot was determined using Image Lab 3.0 software (Bio-Rad Laboratories, Inc.) for the chemiluminescence instrument and ImageJ 1.52t software (National Institutes of Health).

Hepatic stellate cell (HSC) culture. HSC lines were purchased from Sangon Biotech Co., Ltd. and cultured in DMEM
(HyClone; GE Healthcare Life Sciences) supplemented with 10\% FBS (HyClone; GE Healthcare Life Sciences) and $1 \%$ penicillin-streptomycin at $37^{\circ} \mathrm{C}$ in a cell incubator with $5 \% \mathrm{CO}_{2}$. HSCs $\left(3 \times 10^{5} / \mathrm{cm}^{2}\right)$ in the hypoxia/reoxygenation (HR) group were seeded on a 6 -well plate and incubated at $37^{\circ} \mathrm{C}$ for $24 \mathrm{~h}$. After treatment with or without $10 \mu \mathrm{M}$ MAPK inhibitor U0126 (MedChemExpress) at room temperature for $5 \mathrm{~min}$, cells were placed in $94 \% \mathrm{~N}_{2}, 5 \% \mathrm{CO}_{2}$ and $1 \% \mathrm{O}_{2}$ humidified culture incubator for $6 \mathrm{~h}$ at $37^{\circ} \mathrm{C}$, followed by reoxygenation with $5 \% \mathrm{CO}_{2}$ and $95 \%$ air for $12 \mathrm{~h}$ until harvest. Cells in the Con group were cultured in a cell incubator with $5 \% \mathrm{CO}_{2}$ (normoxic conditions) and harvested at the same time as the experimental group $(18 \mathrm{~h})$.

Statistical analysis. Data are presented as the mean \pm SEM ( $n=6 /$ group). Data were analyzed using one-way ANOVA followed by Tukey's post hoc test using SPSS 18.0 (SPSS, Inc.). $\mathrm{P}<0.05$ was considered to indicate a statistically significant difference.

\section{Results}

CIH induces liver fibrosis in rats. To investigate the effects of $\mathrm{CIH}$ exposure in rats, Sirius red staining was used to evaluate liver histological structure. It was identified that 4 weeks of $\mathrm{CIH}$ exposure induced a substantial amount of collagen fibers around the macrovesicular and microvesicular structures, while collagen fibers were rarely detected in the Con group (Fig. 1A and B). When the gene expression of TLR4 was knocked down by TLR4 shRNA lentivirus, liver fibrosis was alleviated (Fig. 1B and D; CIH-TLR4 group vs. CIH group). Furthermore, the results indicated that there was no difference between the $\mathrm{CIH}$-Vector group and $\mathrm{CIH}$ group (Fig. 1B and C). Therefore, it was speculated that 4 weeks of $\mathrm{CIH}$ can induce liver fibrosis in rats and this condition may be associated with TLR4 protein expression.

CIH induces liver inflammation in rats. Previous studies have reported that systemic inflammation is a primary cause of myocardium fibrosis (11). Therefore, the present study examined whether $\mathrm{CIH}$-induced liver fibrosis in rats was associated with inflammation. RT-qPCR and immunohistochemistry were used to detect the expression levels of inflammatory cytokines in the liver of the model animals. RT-qPCR (Fig. 2), immunohistochemical (Fig. 3) and immunohistochemical image analysis (Fig. 4) demonstrated that, compared with Con group, the livers of the $\mathrm{CIH}$ and $\mathrm{CIH}-$ Vector groups had significantly higher expression levels of IL-1 $\beta$, IL-8, MCP-1, TNF- $\alpha$, ICAM-1 and VCAM-1 ( $\mathrm{P}<0.01$ vs. Con group). Moreover, it was found that these effects were reversed by TLR4 shRNA lentivirus treatment $(\mathrm{P}<0.05$ or $\mathrm{P}<0.01$ vs. $\mathrm{CIH}$ group). However, no significant differences were found between the CIH-Vector group and CIH group. Thus, the results suggested that inflammation may play an important role in $\mathrm{CIH}$ exposure-induced liver fibrosis.

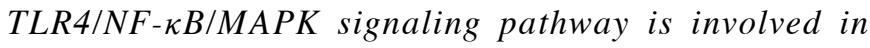
$C I H$-induced liver fibrosis. To determine whether the TLR4/NF- $\kappa$ B/MAPK signaling pathway was involved in $\mathrm{CIH}$-induced liver fibrosis, TLR4 and NF- $\mathrm{NB}$ expression 

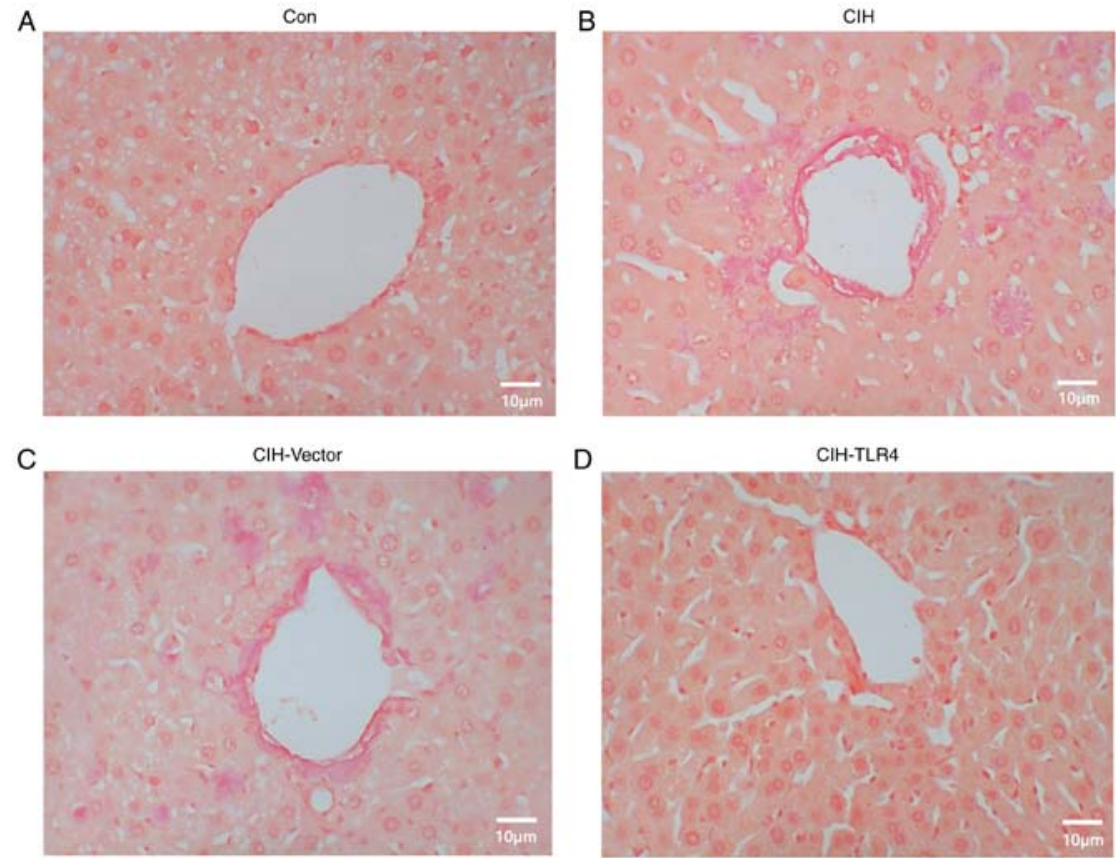

Figure 1. CIH-induced liver fibrosis. After exposing rats to $\mathrm{CIH}$ for 4 weeks, liver tissues were harvested and subjected to Sirius red staining. (A) Con group, (B) CIH group, (C) CIH-Vector and (D) CIH-TLR4 group. Representative images of immunohistochemistry. Magnification, $\mathrm{x} 400$. Con, control; CIH, chronic intermittent hypoxia; CIH-Vector group, $\mathrm{CIH}+$ empty vector lentivirus; CIH-TLR4 group, CIH + TLR4 shRNA lentivirus; shRNA, short hairpin RNA; TLR4, Toll-like receptor 4.
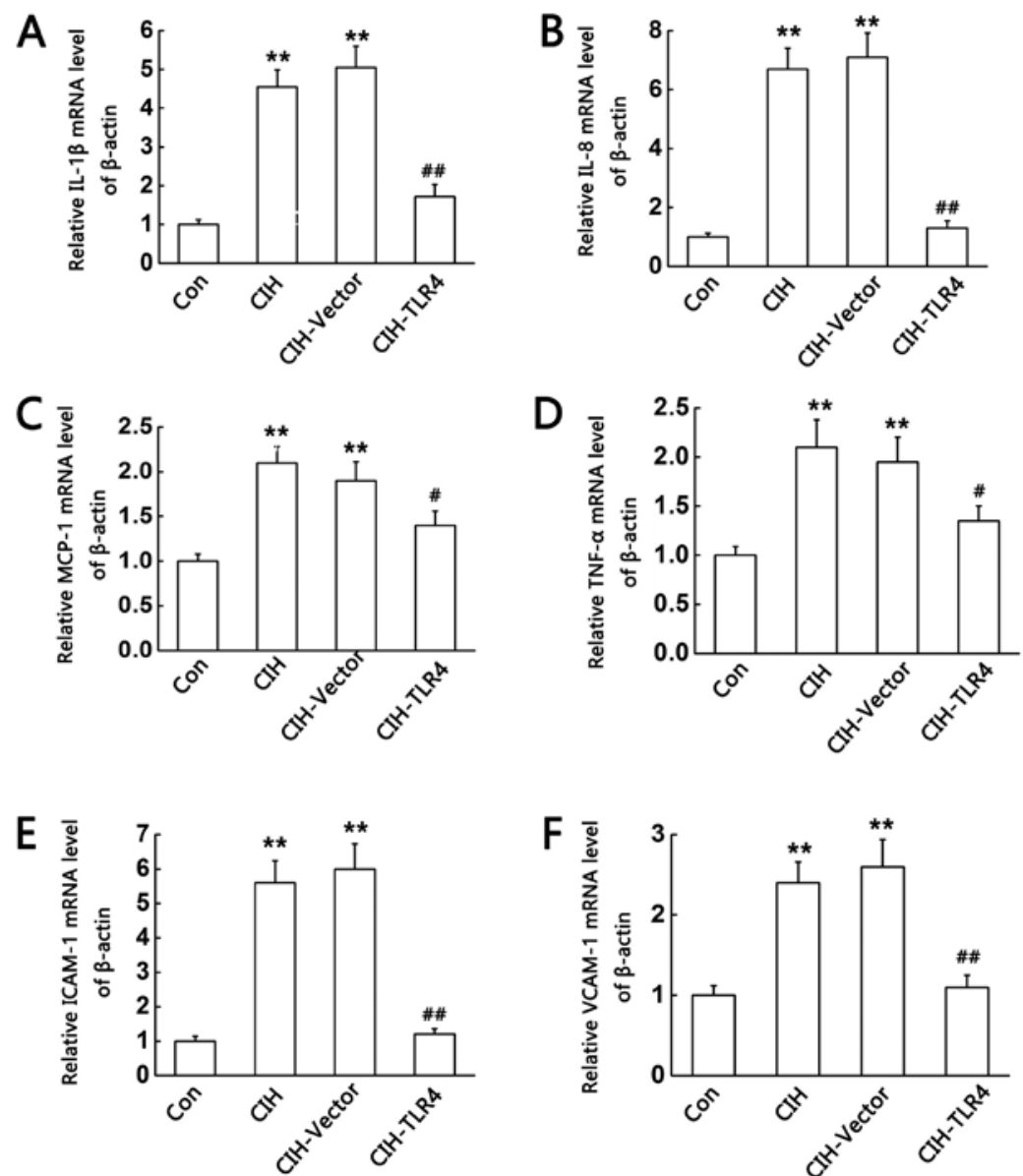

Figure 2. CIH-induced liver mRNA expression levels of IL-1 $\beta$, IL-8, MCP-1, TNF- $\alpha$, ICAM-1 and VCAM-1. After exposing rats to CIH for 4 weeks, liver tissues were harvested and subjected to reverse transcription-quantitative PCR. (A) IL-1 $\beta$, (B) IL-8, (C) MCP-1, (D) TNF- $\alpha$, (E) ICAM-1 and (F) VCAM-1 expression levels. ${ }^{* *} \mathrm{P}<0.01$ vs. control; ${ }^{*} \mathrm{P}<0.05,{ }^{\# \#} \mathrm{P}<0.01$ vs. CIH. Con, control; $\mathrm{CIH}$, chronic intermittent hypoxia; CIH-Vector group, CIH + empty vector lentivirus; CIH-TLR4 group, CIH + TLR4 shRNA lentivirus; shRNA, short hairpin RNA; TLR4, Toll-like receptor 4; IL, interleukin; TNF, tumor necrosis factor; MCP-1, monocyte chemotactic-1; ICAM-1, intercellular adhesion molecule-1; VCAM-1, vascular cell adhesion molecule-1. 
A

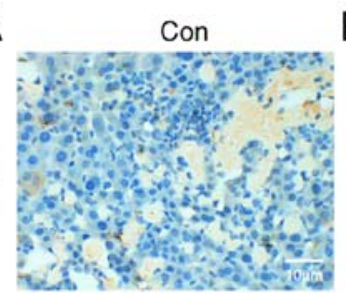

IL-8
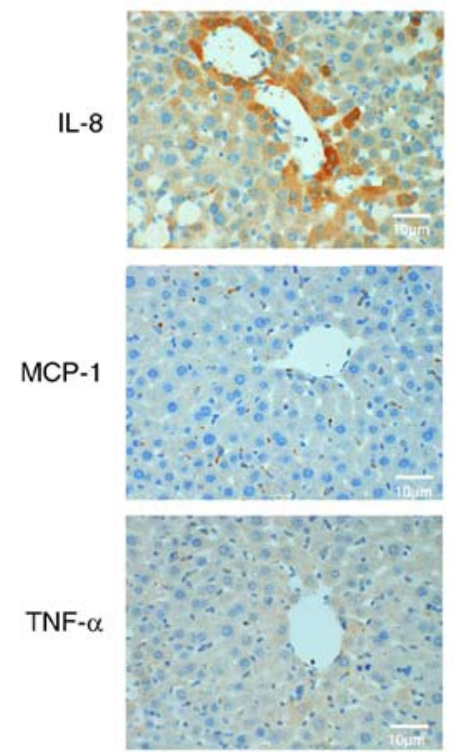

ICAM-1

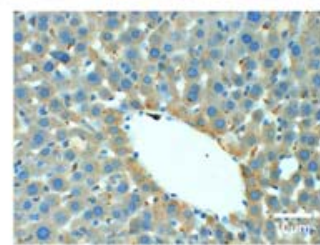

VCAM-1

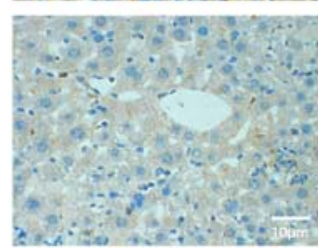

B
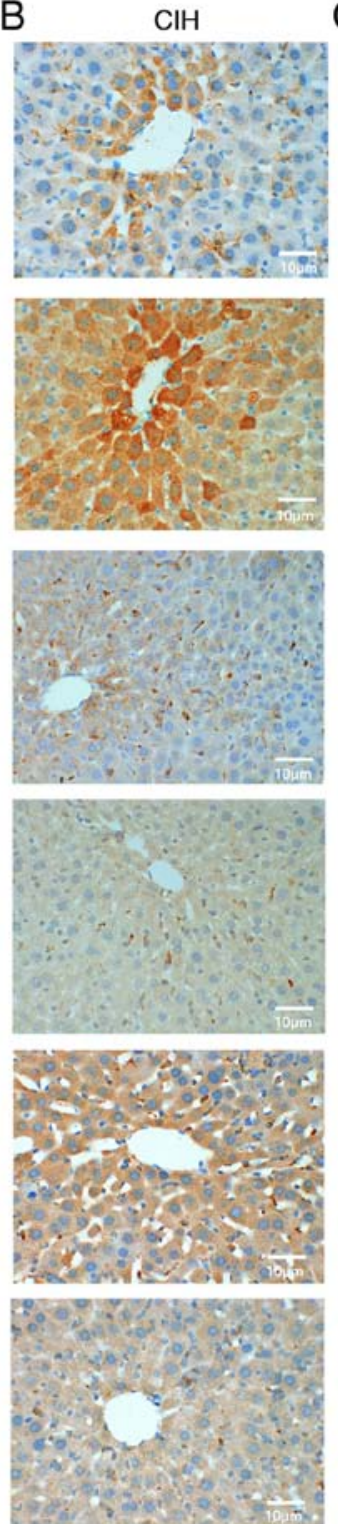
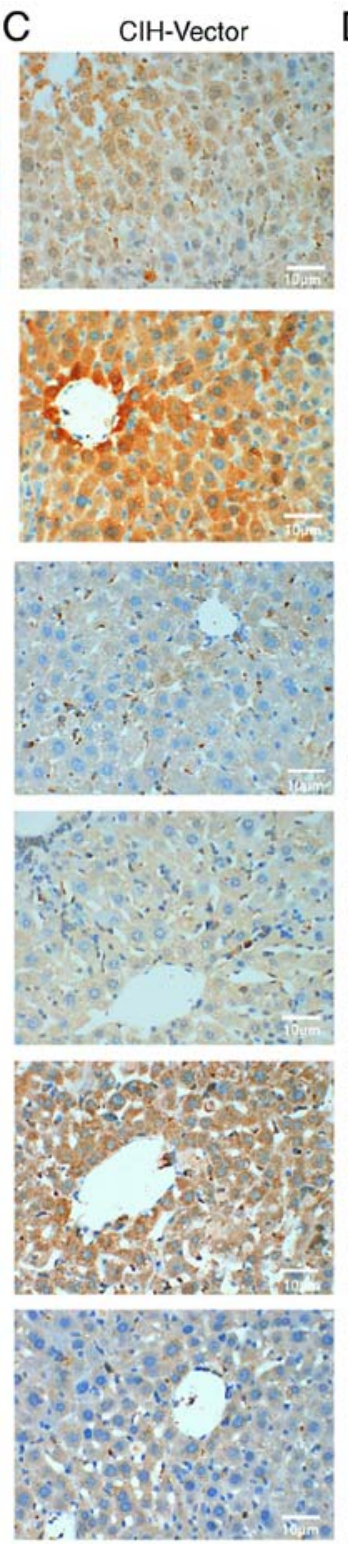
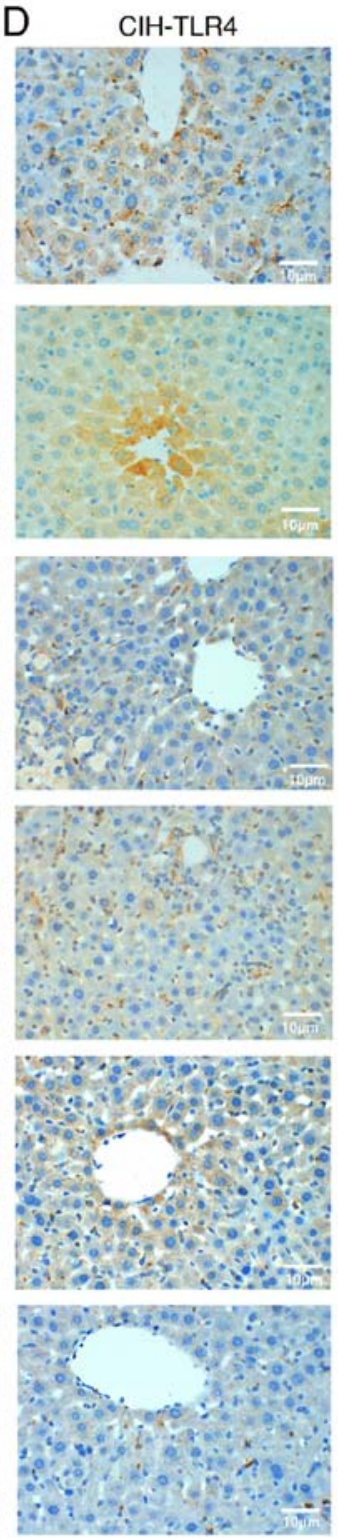

Figure 3. CIH-induced liver inflammation. After exposing rats to CIH for 4 weeks, liver tissues were harvested and subjected to immunohistochemistry staining with antibodies of IL-1 $\beta$, IL-8, MCP-1, TNF- $\alpha$, ICAM-1 and VCAM-1. (A) Con group, (B) CIH group, (C) CIH-Vector group and (D) CIH-TLR4 group. Representative images of immunohistochemistry assay. Magnification, x400. Con, control; CIH, chronic intermittent hypoxia; CIH-Vector group, CIH + empty vector lentivirus; CIH-TLR4 group, CIH + TLR4 shRNA lentivirus; shRNA, short hairpin RNA; TLR4, Toll-like receptor 4; IL, interleukin; TNF, tumor necrosis factor; MCP-1, monocyte chemotactic-1; ICAM-1, intercellular adhesion molecule-1; VCAM-1, vascular cell adhesion molecule-1.

levels were measured by immunohistochemistry and western blot analysis in rats. It was identified that TLR4 and $\mathrm{NF}-\kappa \mathrm{B}$ expression levels were significantly increased in the $\mathrm{CIH}$ and CIH-Vector groups (Fig. 5; $\mathrm{P}<0.01$ vs. Con group), while TLR4 shRNA lentivirus treatment decreased the expression levels of these proteins (Fig. 5; $\mathrm{P}<0.01$ vs. $\mathrm{CIH}$ group).

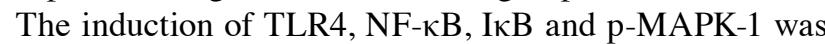
assessed by western blot analysis of HSCs. It was demonstrated that the protein expression levels of TLR4, NF- $\mathrm{B}$, I $\kappa \mathrm{B}$ and $\mathrm{p}-\mathrm{MAPK}-1$ in the hypoxia/reoxygenation (HR) group were significantly higher compared with the Con group $(\mathrm{P}<0.01$; Fig. 6). Furthermore, these effects were significantly reversed by application of U0126, a type of MAPK inhibitor. Collectively, the results indicated the involvement of the TLR4/NF- $\mathrm{B} / \mathrm{MAPK}$ signaling pathway in CIH-induced liver fibrosis.

\section{Discussion}

Numerous animal models used in the study of hypoxia have been developed over the past years, of which the most widely used is the CIH model, which simulates the intermittent hypoxia factor of OSAS $(3,8)$. There are several different methods to verify the successful establishment of the OSAS model, such as monitoring the electroencephalogram, oronasal air flow, dynamic blood oxygen for $2 \mathrm{~h}$, mean blood oxygen saturation, minimum blood oxygen saturation and sleep apnea index (6). In the present study, a CIH model was established and used to study the underlying mechanisms of liver fibrosis in OSAS. It was found that 4 weeks of $\mathrm{CIH}$ exposure induced distinct hepatic fibrosis around the macrovesicular and microvesicular structures. Moreover, it was demonstrated that knockdown of TLR4 using TLR4 shRNA lentivirus resulted in alleviated 
A

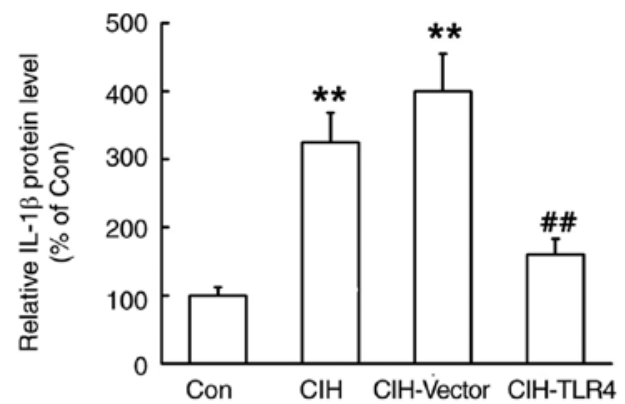

C

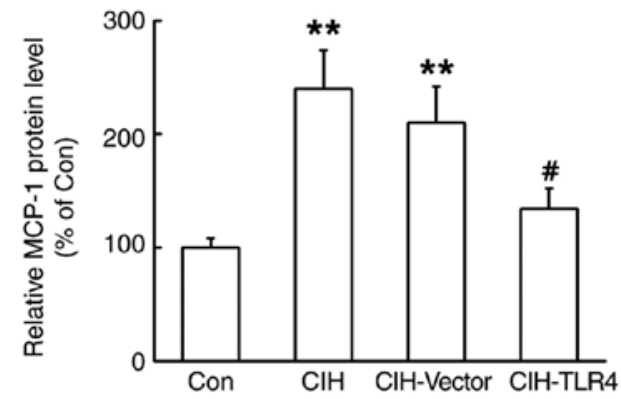

E

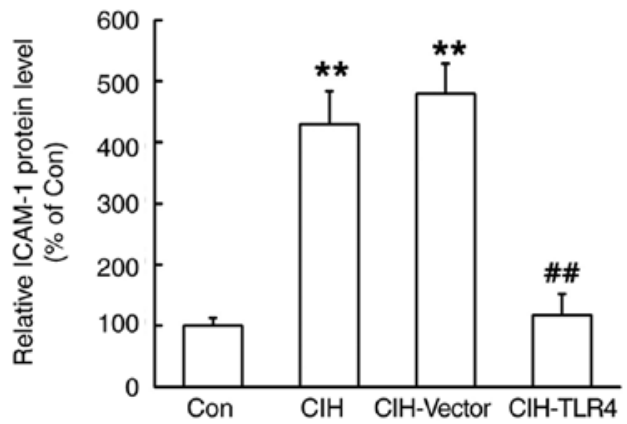

B
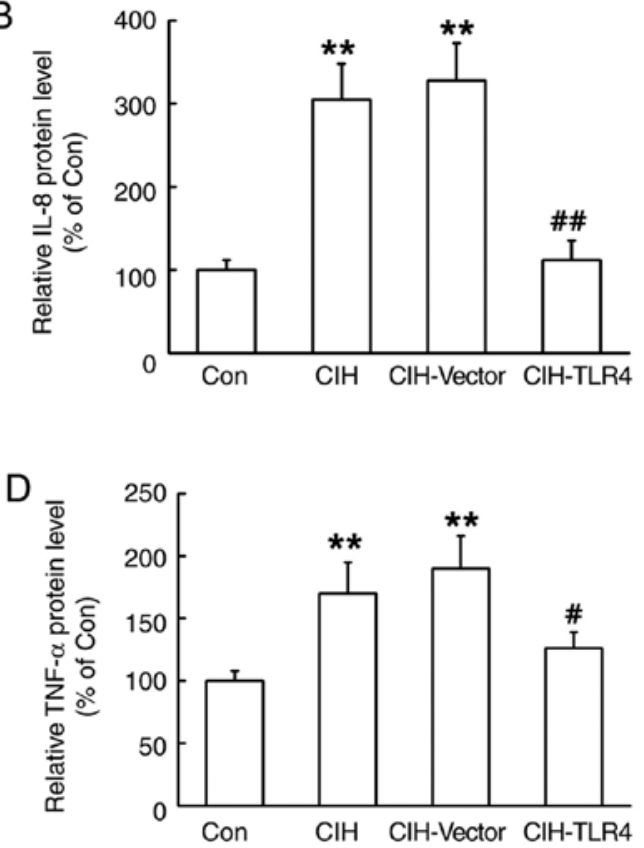

F

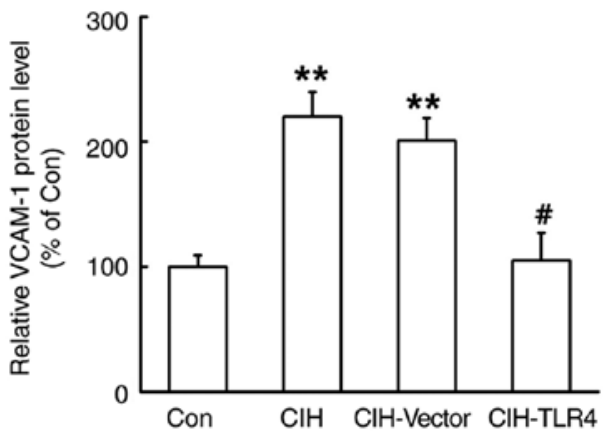

Figure 4. Statistical results of immunohistochemical images. After exposing rats to CIH for 4 weeks, liver tissues were harvested and subjected to immunohistochemistry staining with antibodies of IL-1 $\beta$, IL-8, MCP-1, TNF- $\alpha$, ICAM-1 and VCAM-1. (A) IL-1 $\beta$, (B) IL-8, (C) MCP-1, (D) TNF- $\alpha$, (E) ICAM-1 and (F) VCAM-1 expression levels. ${ }^{* *} \mathrm{P}<0.01$ vs. control; ${ }^{\#} \mathrm{P}<0.05,{ }^{\# \#} \mathrm{P}<0.01$ vs. CIH. Con, control; CIH, chronic intermittent hypoxia; CIH-Vector group, CIH + empty vector lentivirus; CIH-TLR4 group, CIH + TLR4 shRNA lentivirus; shRNA, short hairpin RNA; TLR4, Toll-like receptor 4; IL, interleukin; TNF, tumor necrosis factor; MCP-1, monocyte chemotactic-1; ICAM-1, intercellular adhesion molecule-1; VCAM-1, vascular cell adhesion molecule-1.

liver fibrosis. Immunohistochemical results also identified that liver samples of the $\mathrm{CIH}$ and $\mathrm{CIH}-$ Vector groups presented an inflammatory state with increased expression levels of IL-1 $\beta$, IL-8, MCP-1, TNF- $\alpha$, ICAM-1 and VCAM-1, which could be reversed by TLR4 shRNA lentivirus treatment. These results were consistent with previous findings reporting that patients with OSAS have increased systemic inflammation (2), which contributes to liver fibrosis and may be alleviated by inhibiting TLR4 expression.

Previous studies have shown that OSAS is an inflammatory state characterized by increases in the levels of circulating biomarkers of inflammation $(2,26)$. While the inflammatory mechanism underlying OSAS has not been fully elucidated, it has been observed that the increased inflammation is partly mediated by HR $(26,27)$. Savransky et al $(28)$ revealed that $\mathrm{CIH}$ is a potent effective proinflammatory cytokine that not only induces hyperglycemia and liver lipid peroxidation, but also enhances the activity of NF- $\kappa \mathrm{B}$, which is the main regulator of the inflammatory response. In addition, it has been observed that patients with OSAS exhibit significantly increased serum NF- $\kappa \mathrm{B}$ activities (2). Furthermore, serum levels of multiple
$\mathrm{NF}-\kappa \mathrm{B}$-dependent proinflammatory cytokine and adhesion molecules, such as IL-1 $\beta$, IL-6, IL-8, TNF- $\alpha$, MCP-1 and VCAM-1, are also elevated in patients with OSAS $(29,30)$. Moreover, Aron-Wisnewsky et al (8) reported that $\mathrm{CIH}$ is strongly associated with increased systemic inflammatory responses, as well as with more serious fibrosis or inflammatory liver injuries. The present results suggested that 4 weeks of $\mathrm{CIH}$ exposure induced liver fibrosis in the $\mathrm{CIH}$ and $\mathrm{CIH}-$ Vector groups, while the $\mathrm{CIH}-$ Vector group presented an inflammatory state with increased expression of IL-1 $\beta$, IL-8, MCP-1, TNF- $\alpha$, ICAM-1 and VCAM-1. NF- $\kappa$ B is considered to be an oxidant-sensitive transcription factor, and activation of NF- $\mathrm{NB}$ promotes inflammation and multiple tissue injury in response to $\mathrm{CIH}$ and in liver disease conditions $(31,32)$. Furthermore, the present results indicated that 4 weeks of $\mathrm{CIH}$ exposure increased $\mathrm{NF}-\kappa \mathrm{B}$ and TLR4 expression levels in the liver. In addition, it was found that protein expression levels of TLR4, NF- $\kappa \mathrm{B}, \mathrm{I} \kappa \mathrm{B}$ and p-MAPK-1 in the HR group were significantly higher compared with the Con group, and these effects could be reversed by application of the MAPK inhibitor U0126. However, a limitation of the present study 

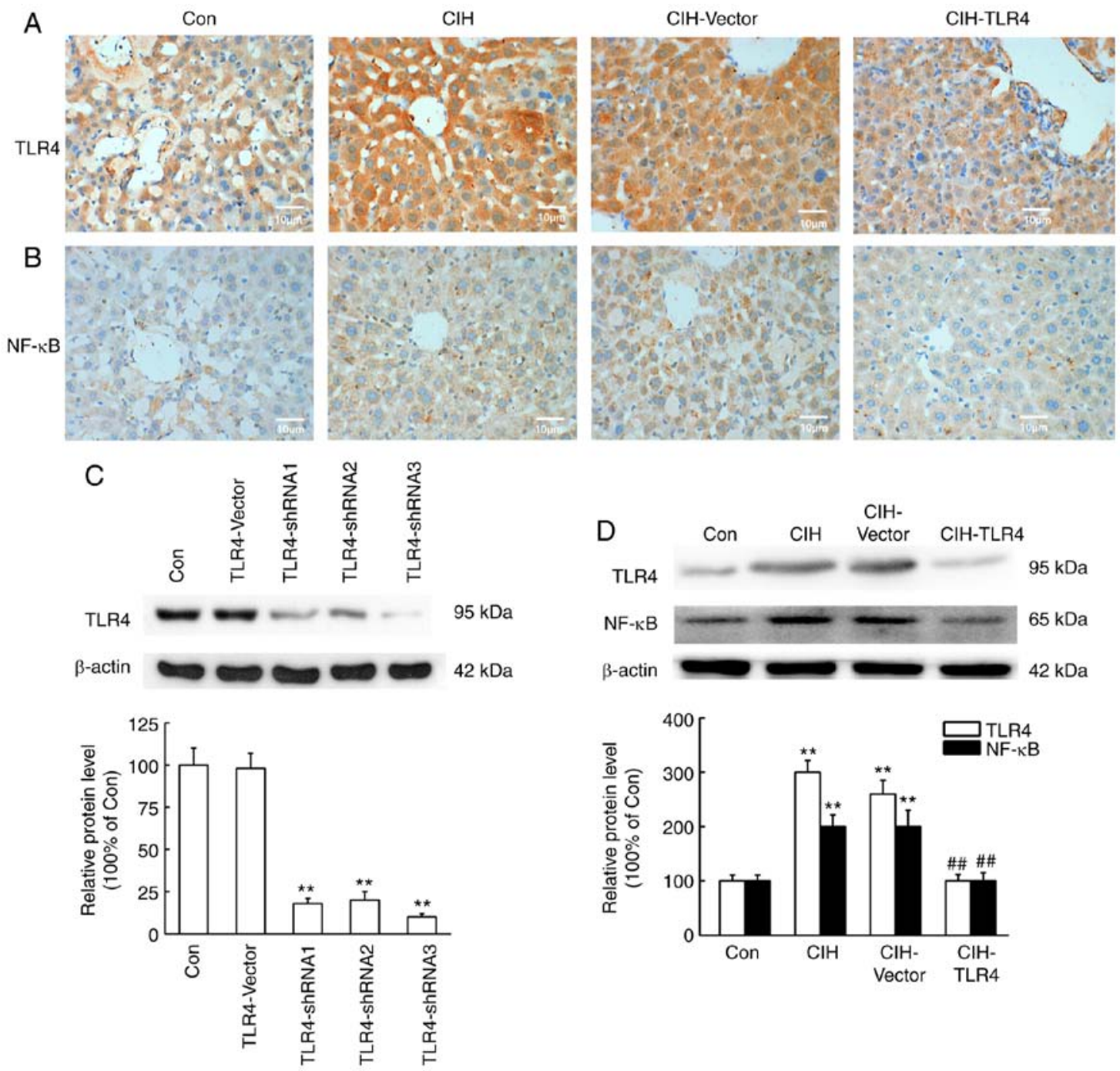

Figure 5. TLR4 shRNA lentivirus treatment decreases the expression levels of TLR4 and NF- $\mathrm{B}$. After exposing rats to CIH for 4 weeks, liver tissues were harvested and subjected to immunohistochemistry staining and western blotting. Immunohistochemistry results of (A) TLR4 and (B) NF- $\kappa$ B. Protein expres-

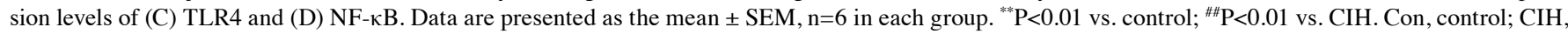
chronic intermittent hypoxia; CIH-Vector group, CIH + empty vector lentivirus; CIH-TLR4 group, CIH + TLR4 shRNA lentivirus; shRNA, short hairpin RNA; TLR4, Toll-like receptor 4; TNF, tumor necrosis factor.

was that $\mathrm{p}-\mathrm{NF}-\kappa \mathrm{B}$ was not detected; $\mathrm{p}-\mathrm{NF}-\kappa \mathrm{B}$ is the activated state of $N F-\kappa B$, thus it would be beneficial to detect the effects

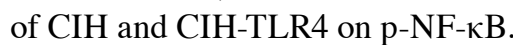

$\mathrm{CIH}$ can induce an unbalanced production of large amounts of reactive oxygen species (ROS) and endogenous antioxidant defense mechanisms, which enhance oxidative stress $(33,34)$. ROS can also activate nuclear transcriptional factors, including $\mathrm{NF}-\kappa \mathrm{B}$ and hypoxia-inducible factors (HIFs), which in turn promote the production of inflammatory cytokines, such as IL-1 $\beta$ and IL-8, as observed in a CIH model $(35,36)$. HIFs are the main transcriptional regulators of the hypoxia response, and belong to a family of heterodimeric transcription factors (37). Furthermore, in almost all tissues and cells, HIFs act as the main regulator of the body to maintain homeostasis in response to hypoxia (38). Activated HIFs can be rapidly transferred to the nucleus, where they bind to hypoxia response elements of the target gene promoter region to regulate gene transcription $(37,39)$. Previous studies have revealed that activation of HIF1 $\alpha$ plays a key role in downstream signaling transduction of lipopolysaccharide (LPS) stimulation via the pattern recognition receptor TLR4. For example, LPS can upregulate HIF1 $\alpha$ expression in rat liver, thus upregulating the expression of aldolase, a type of HIF1 $\alpha$ target gene $(40,41)$. In addition, it has been shown that the activity of LPS-induced HIF1 $\alpha$ mainly depends on $\mathrm{NF}-\kappa \mathrm{B}$, the inflammatory master regulator of a group of proteins, which is predominantly regulated by inhibiting the transcriptional action of $\mathrm{I} \kappa \mathrm{B}(38,42)$.

Clinical and animal studies have reported that the expression of TLR4 is closely related to activation of inflammatory response in patients with OSAS $(20,22)$. Moreover, TLR4 is a typical pattern recognition receptor located upstream of $\mathrm{NF}-\kappa \mathrm{B}$, and is closely related to the activation of inflammatory responses mainly via MyD88- and TIR-domain-containing adapter-inducing interferon- $\beta$-dependent pathways (43). It has also been revealed that activation of the TLR4/MyD88 signaling pathway in HR is positively correlated with myocardial injury in animal models (44). Shimamoto et al (45) observed that myocardial ischemia activates the TLR4/MyD88-dependent signal pathway and increases the activation of $\mathrm{NF}-\kappa \mathrm{B}$, which ultimately leads to the release of 
A

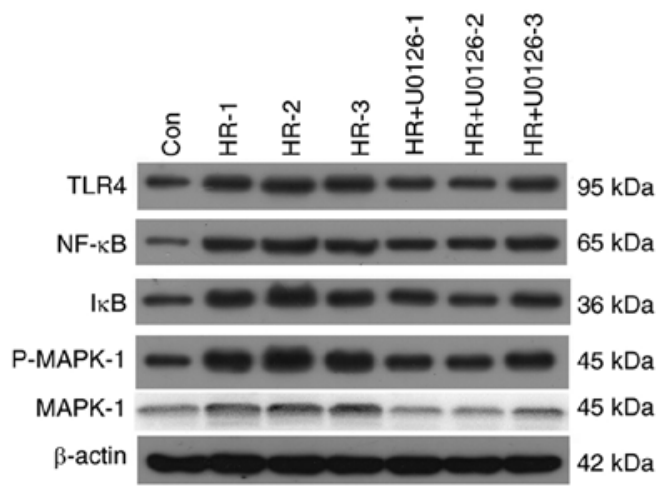

B

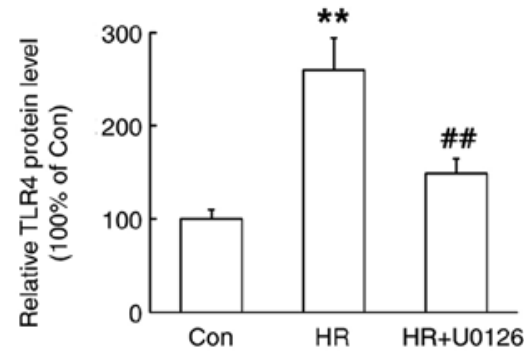

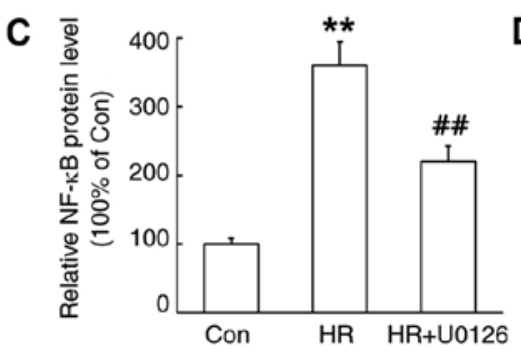
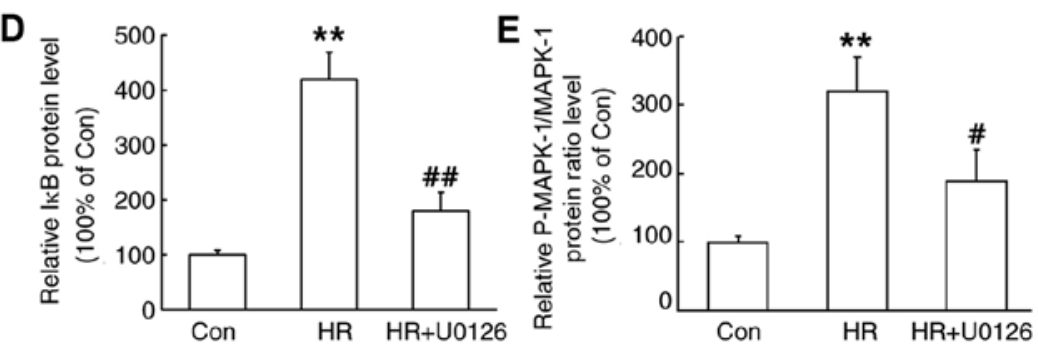

Figure 6. Effects of HR and MAPK inhibitor U0126 on the expression levels of TLR4, NF-кB, IкB and p-MAPK-1 in HSCs. After treating hepatic stellate cells with or without MAPK inhibitor U0126 $(10 \mu \mathrm{M})$, cells were placed in a 94\% $\mathrm{N}_{2}, 5 \% \mathrm{CO}_{2}$ and $1 \% \mathrm{O}_{2}$ humidified culture incubator for $6 \mathrm{~h}$, followed by reoxygenation with $5 \% \mathrm{CO}_{2}$ and $95 \%$ air for $12 \mathrm{~h}$. Protein expression was determined by western blot analysis. (A) Representative protein bands. Protein expression levels of (B) TLR4, (C) NF-kB, (D) IкB and (E) p-MAPK-1. Data are presented as the mean \pm SEM, $n=6$ in each group. ${ }^{* *} \mathrm{P}<0.01$ vs. control;

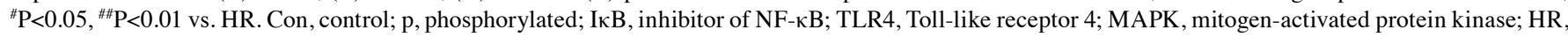
hypoxia/reoxygenation.

innate cytokines in the heart. Furthermore, Takahashi et al (46) showed that healthy mice and TSK mouse fibrosis models exhibited notable fibrosis after bleomycin treatment, while TLR4 knockout mice were protected from fibrosis, thus indicating that TLR4 plays an important role in the fiberization process. Moreover, non-functional TLR4 mutations or TLR4 knockout can effectively protect mice against the development of renal tissue dysfunction, inflammatory damage and fibrosis in a model of chronic kidney injury $(15,16)$. It was found that similar injury also occurred in the rat liver in the present study, and this condition could be significantly alleviated by knocking down TLR4 Therefore, it was speculated that TLR4 and NF- $\mathrm{BB}$ mediated $\mathrm{CIH}$-induced inflammation and liver fibrosis.

In conclusion, the preliminary results indicated that $\mathrm{CIH}$ could induce liver fibrosis in rats, and this effect was positively associated with inflammation and the TLR4/NF- $\mathrm{B} / \mathrm{MAPK}$ signaling pathway. Furthermore, it was demonstrated that the expression of TLR4 was associated with the pathogenesis of liver fibrosis in $\mathrm{CIH}$; thus, the development of a novel method that inhibits TLR4 expression may be a viable strategy for clinical prevention of liver fibrosis in patients with OSAS.

\section{Acknowledgements}

Not applicable.

\section{Funding}

This work was supported by Natural Science Foundation of Fujian Province (grant nos. 2018J0105, 2018Y0032 and 2016J01441) and Young and Middle-aged Backbone Key Research Project of Fujian Province (grant no. 2016ZQN55).

\section{Availability of data and materials}

The datasets used and/or analyzed during the current study are available from the corresponding author on reasonable request.

\section{Authors' contributions}

ZPL, XPY, YJZ and SYC conducted the experiments and analyzed the data. HLL conceived and supervised the project, contributed to the design of the experiments, discussed the data and wrote the manuscript with contributions from ZPL, XPY, YJZ and SYC. All authors read and approved the final manuscript.

\section{Ethics approval and consent to participate}

All animal procedures were approved by the Ethical Committee of Experimental Animals of Fujian Medical University and The Second Affiliated Hospital of Fujian Medical University. Animal experiments were carried out in accordance with animal welfare requirements and the Guidelines for the Care and Use of Laboratory Animals published by the P.R. China Ministry of Health.

\section{Patient consent for publication}

Not applicable. 


\section{Competing interests}

The authors declare that they have no competing interests.

\section{References}

1. Philip P, Bioulac S, Altena E, Morin CM, Ghorayeb I, Coste O, Monteyrol PJ and Micoulaud-Franchi JA: Specific insomnia symptoms and self-efficacy explain CPAP compliance in a sample of OSAS patients. PLoS One 13: e0195343, 2018.

2. Paschetta E, Belci P, Alisi A, Liccardo D, Cutrera R, Musso G and Nobili V: OSAS-related inflammatory mechanisms of liver injury in nonalcoholic fatty liver disease. Mediators Inflamm 2015: 815721, 2015.

3. Arnold J, Sunilkumar M, Krishna V, Yoganand SP, Kumar MS and Shanmugapriyan D: Obstructive sleep apnea. J Pharm Bioallied Sci 9 (Suppl 1): S26-S28, 2017.

4. O'Halloran KD, Lewis P and McDonald F: Sex, stress and sleep apnoea: Decreased susceptibility to upper airway muscle dysfunction following intermittent hypoxia in females. Respir Physiol Neurobiol 245: 76-82, 2017.

5. Kang NY, Ivanovska J, Tamir-Hostovsky L, Belik J and Gauda EB: Chronic intermittent hypoxia in premature infants: The link between low fat stores, adiponectin receptor signaling and lung injury. Adv Exp Med Biol 1071: 151-157, 2018.

6. Chen Q, Lin G, Huang J, Chen G, Huang X and Lin Q: Expression profile of long non-coding RNAs in rat models of OSA-induced cardiovascular disease: New insight into pathogenesis. Sleep Breath 23: 795-804, 2019.

7. Coelho NR, Dias CG, João Correia M, Grácio P, Serpa J, Monteiro EC, Diogo LN and Pereira SA: Cysteine oxidative dynamics underlies hypertension and kidney dysfunction induced by chronic intermittent hypoxia. Adv Exp Med Biol 1071: 83-88, 2018.

8. Aron-Wisnewsky J, Clement K and Pépin JL: Nonalcoholic fatty liver disease and obstructive sleep apnea. Metabolism 65 : $1124-1135,2016$.

9. Sozer V, Kutnu M, Atahan E, Caliskaner Ozturk B, Hysi E, Cabuk C, Musellim B, Simsek G and Uzun H: Changes in inflammatory mediators as a result of intermittent hypoxia in obstructive sleep apnea syndrome. Clin Respir J 12: 1615-1622, 2018.

10. Wu CX, Liu Y and Zhang JC: Chronic intermittent hypoxia and hypertension: A review of systemic inflammation and Chinese medicine. Chin J Integr Med 19: 394-400, 2013.

11. Ryan S and McNicholas WT: Inflammatory cardiovascular risk markers in obstructive sleep apnoea syndrome. Cardiovasc Hematol Agents Med Chem 7: 76-81, 2009.

12. Drager LF, Tavoni TM, Silva VM, Santos RD, Pedrosa RP, Bortolotto LA, Vinagre CG, Polotsky VY, Lorenzi-Filho G and Maranhao RC: Obstructive sleep apnea and effects of continuous positive airway pressure on triglyceride-rich lipoprotein metabolism. J Lipid Res 59: 1027-1033, 2018.

13. Ropert C: How toll-like receptors reveal monocyte plasticity: The cutting edge of antiinflammatory therapy. Cell Mol Life Sci 76: 745-755, 2019 .

14. Mohan S and Gupta D: Crosstalk of toll-like receptors signaling and Nrf2 pathway for regulation of inflammation. Biomed Pharmacother 108: 1866-1878, 2018.

15. Chowdhury P, Sacks SH and Sheerin NS: Toll-like receptors TLR2 and TLR4 initiate the innate immune response of the renal tubular epithelium to bacterial products. Clin Exp Immunol 145 : 346-356, 2006

16. González-Guerrero C, Cannata-Ortiz P, Guerri C, Egido J, Ortiz A and Ramos AM: TLR4-mediated inflammation is a key pathogenic event leading to kidney damage and fibrosis in cyclosporine nephrotoxicity. Arch Toxicol 91: 1925-1939, 2017.

17. Mukherjee S, Karmakar S and Babu SP: TLR2 and TLR4 mediated host immune responses in major infectious diseases: A review. Braz J Infect Dis 20: 193-204, 2016.

18. Zhou M, Xu W, Wang J, Yan J, Shi Y, Zhang C, Ge W, Wu J, Du P and Chen Y: Boosting mTOR-dependent autophagy via upstream TLR4-MyD88-MAPK signalling and downstream NF- $\kappa \mathrm{B}$ pathway quenches intestinal inflammation and oxidative stress injury. EBioMedicine 35: 345-360, 2018.

19. $\mathrm{Hu} \mathrm{H}$ and Li H: Prunetin inhibits lipopolysaccharide-induced inflammatory cytokine production and MUC5AC expression by inactivating the TLR4/MyD88 pathway in human nasal epithelial cells. Biomed Pharmacother 106: 1469-1477, 2018.
20. Yuan X, Deng Y, Guo X, Shang J, Zhu D and Liu H: Atorvastatin attenuates myocardial remodeling induced by chronic intermittent hypoxia in rats: Partly involvement of TLR-4/MYD88 pathway. Biochem Biophys Res Commun 446: 292-297, 2014.

21. Deng Y, Yuan X, Guo XL, Zhu D, Pan YY and Liu HG: Efficacy of atorvastatin on hippocampal neuronal damage caused by chronic intermittent hypoxia: Involving TLR4 and its downstream signaling pathway. Respir Physiol Neurobiol 218: 57-63, 2015.

22. Viciani E, Montagnani F, Tavarini S, Tordini G, Maccari S, Morandi M, Faenzi E, Biagini C, Romano A, Salerni L, et al: Paediatric obstructive sleep apnoea syndrome (OSAS) is associated with tonsil colonisation by Streptococcus pyogenes. Sci Rep 6: 20609, 2016

23. Dong Z, Shang H, Chen YQ, Pan LL, Bhatia M and Sun J: Sulforaphane protects pancreatic acinar cell injury by modulating Nrf2-Mediated oxidative stress and NLRP3 inflammatory pathway. Oxid Med Cell Longev 2016: 7864150, 2016.

24. Livak KJ and Schmittgen TD: Analysis of relative gene expression data using real-time quantitative PCR and the 2(-Delta Delta C(T)) method. Methods 25: 402-408, 2001.

25. Zeng X, Guo R, Dong M, Zheng J, Lin H and Lu H: Contribution of TLR4 signaling in intermittent hypoxia-mediated atherosclerosis progression. J Transl Med 16: 106, 2018.

26. Ryan S, Taylor CT and McNicholas WT: Selective activation of inflammatory pathways by intermittent hypoxia in obstructive sleep apnea syndrome. Circulation 112: 2660-2667, 2005.

27. Ye J, Liu H, Li Y, Liu X and Zhu JM: Increased serum levels of C-reactive protein and matrix metalloproteinase-9 in obstructive sleep apnea syndrome. Chin Med J (Engl) 120: 1482-1486, 2007.

28. Savransky V, Bevans S, Nanayakkara A, Li J, Smith PL, Torbenson MS and Polotsky VY: Chronic intermittent hypoxia causes hepatitis in a mouse model of diet-induced fatty liver. Am J Physiol Gastrointest Liver Physiol 293: G871-G877, 2007.

29. de Lima FF, Mazzotti DR, Tufik S and Bittencourt L: The role inflammatory response genes in obstructive sleep apnea syndrome: A review. Sleep Breath 20: 331-338, 2016.

30. Tamaki S, Yamauchi M, Fukuoka A, Makinodan K, Koyama N, Tomoda K, Yoshikawa M and Kimura H: Production of inflammatory mediators by monocytes in patients with obstructive sleep apnea syndrome. Intern Med 48: 1255-1262, 2009.

31. Wang H, Wang Y, Xia T, Liu Y, Liu T, Shi X and Li Y: Pathogenesis of abnormal hepatic lipid metabolism induced by chronic intermittent hypoxia in rats and the therapeutic effect of N-acetylcysteine. Med Sci Monit 24: 4583-4591, 2018.

32. Song D, Fang G, Mao SZ, Ye X, Liu G, Gong Y and Liu SF: Chronic intermittent hypoxia induces atherosclerosis by NF- $\kappa$ B-dependent mechanisms. Biochim Biophys Acta 1822: $1650-1659,2012$

33. Morgan BJ, Bates ML, Rio RD, Wang Z and Dopp JM: Oxidative stress augments chemoreflex sensitivity in rats exposed to chronic intermittent hypoxia. Respir Physiol Neurobiol 234: 47-59, 2016

34. Lüneburg N, Siques P, Brito J, Arriaza K, Pena E, Klose H, Leon-Velarde $\mathrm{F}$ and Böger RH: Long-Term chronic intermittent hypobaric hypoxia in rats causes an imbalance in the asymmetric dimethylarginine/nitric oxide pathway and ROS activity: A possible synergistic mechanism for altitude pulmonary hypertension? Pulm Med 2016: 6578578, 2016.

35. Al-Anazi A, Parhar R, Saleh S, Al-Hijailan R, Inglis A, Al-Jufan M, Bazzi M, Hashmi S, Conca W, Collison K and Al-Mohanna F: Intracellular calcium and NF- $\mathrm{B}$ regulate hypoxia-induced leptin, VEGF, IL-6 and adiponectin secretion in human adipocytes. Life Sci 212: 275-284, 2018.

36. Yang X, Chen GT, Wang YQ, Xian S, Zhang L, Zhu SM, Pan F and Cheng YX: TLR4 promotes the expression of HIF-1 $\alpha$ by triggering reactive oxygen species in cervical cancer cells in vitro-implications for therapeutic intervention. Mol Med Rep 17: 2229-2238, 2018.

37. Masoud GN and Li W: HIF-1 $\alpha$ pathway: Role, regulation and intervention for cancer therapy. Acta Pharm Sin B 5: 378-389, 2015.

38. Ullah K, Rosendahl AH, Izzi V, Bergmann U, Pihlajaniemi T, Mäki JM and Myllyharju J: Hypoxia-inducible factor prolyl-4-hydroxylase-1 is a convergent point in the reciprocal negative regulation of $\mathrm{NF}-\kappa \mathrm{B}$ and $\mathrm{p} 53$ signaling pathways. Sci Rep 7: 17220, 2017.

39. Fu D, He C, Wei J, Zhang Z, Luo Y, Tan H and Ren C: PGK1 is a potential survival biomarker and invasion promoter by regulating the HIF-1 $\alpha$-mediated epithelial-mesenchymal transition process in breast cancer. Cell Physiol Biochem 51: 2434-2444, 2018. 
40. Shirasuna K, Shimamura N, Seno K, Ohtsu A, Shiratsuki S Ohkuchi A, Suzuki H, Matsubara S, Nagayama S, Iwata $H$ and Kuwayama T: Moderate hypoxia down-regulates interleukin-6 secretion and TLR4 expression in human Sw.71 placental cells. Cell Physiol Biochem 36: 2149-2160, 2015.

41. Menon D, Coll R, O'Neill LA and Board PG: GSTO1-1 modulates metabolism in macrophages activated through the LPS and TLR4 pathway. J Cell Sci 128: 1982-1990, 2015.

42. Siegert I, Schodel J, Nairz M, Schatz V, Dettmer K, Dick C, Kalucka J, Franke K, Ehrenschwender M, Schley G, et al: Ferritin-mediated iron sequestration stabilizes hypoxia-inducible factor-1 $\alpha$ upon LPS activation in the presence of ample oxygen. Cell Rep 13: 2048-2055, 2015.

43. Funami K, Matsumoto M, Oshiumi H, Inagaki F and Seya T: Functional interfaces between TICAM-2/TRAM and TICAM-1/TRIF in TLR4 signaling. Biochem Soc Trans 45: 929-935, 2017.
44. Zhang X, Du Q, Yang Y, Wang J, Dou S, Liu C and Duan J: The protective effect of luteolin on myocardial ischemia/reperfusion (I/R) injury through TLR4/NF- $\kappa \mathrm{B} / \mathrm{NLRP} 3$ inflammasome pathway. Biomed Pharmacother 91: 1042-1052, 2017.

45. Shimamoto A, Chong AJ, Yada M, Shomura S, Takayama H, Fleisig AJ, Agnew ML,Hampton CR, Rothnie CL, Spring DJ, et al: Inhibition of Toll-like receptor 4 with eritoran attenuates myocardial ischemia-reperfusion injury. Circulation 114(1 Suppl): I270-I274, 2006.

46. Takahashi T, Asano Y, Ichimura Y, Toyama T, Taniguchi T, Noda S, Akamata K, Tada Y, Sugaya M, Kadono T and Sato S: Amelioration of tissue fibrosis by toll-like receptor 4 knockout in murine models of systemic sclerosis. Arthritis Rheumatol 67: 254-265, 2015.

This work is licensed under a Creative Commons Attribution-NonCommercial-NoDerivatives 4.0 International (CC BY-NC-ND 4.0) License. 